\title{
Neural correlates of recognition memory in children with febrile seizures: evidence from functional magnetic resonance imaging
}

\author{
Kerstin H. Kipp ${ }^{1}$, Bertram Opitz ${ }^{1}$, Martina Becker ${ }^{1}$, Juliane Hofmann ${ }^{1}$, Christoph Krick ${ }^{2}$, Ludwig Gortner ${ }^{3}$ \\ and Axel Mecklinger ${ }^{1}$
}

1 Experimental Neuropsychology Unit, Department of Psychology, Saarland University, Saarbruecken, Germany

${ }^{2}$ Department of Neuroradiology, Saarland University Hospital, Homburg, Germany

${ }^{3}$ Department of Paediatrics, Paediatric Intensive Care Medicine, Saarland University Hospital, Homburg, Germany

\section{Edited by:}

Hans-Jochen Heinze, University of

Magdeburg, Germany

Reviewed by:

Carme Junque, University of

Barcelona, Spain

Alan Richardson-Klavehn, Otto von

Guericke University, Germany

\section{*Correspondence:}

Kerstin H. Kipp, Experimental

Neuropsychology Unit, Department of

Psychology, Saarland University,

D-66123 Saarbrücken, Germany.

e-mail:k.kipp@mx.uni-saarland.de
Febrile seizures (FS) are assumed to not have adverse long-term effects on cognitive development. Nevertheless, FS are often associated with hippocampal sclerosis which can imply episodic memory deficits. This interrelation has hardly been studied so far. In the current study 13 children who had suffered from FS during infancy and 14 control children (7 to 9 -years-old) were examined for episodic and semantic memory with standardized neuropsychological tests. Furthermore, using functional magnetic resonance imaging (fMRI) we studied neuronal activation while the children performed a continuous recognition memory task. The analysis of the behavioral data of the neuropsychological tests and the recognition memory experiment did not reveal any between-group differences in memory performance. Consistent with other studies fMRI revealed repetition enhancement effects for both groups in a variety of brain regions (e.g., right middle frontal gyrus, left parahippocampal gyrus) and a repetition suppression effect in the right superior temporal gyrus. Different neural activation patterns between both groups were obtained selectively within the right supramarginal gyrus (BA 40). In the control group correct rejections of new items were associated with stronger activation than correctly identified old items (HITs) whereas in the FS group no difference occurred. On the background that the right supramarginal gyrus is assumed to mediate a top-down process to internally direct attention toward recollected information, the results could indicate that control children used strategic recollection in order to reject new items (recall-to-reject). In contrast, the missing effect in the FS group could reflect a lack of strategy use, possibly due to impaired recollective processing. This study demonstrates that FS, even with mainly benign courses, can be accompanied by selective modifications in the neural structures underlying recognition memory.

Keywords: memory development, recognition, episodic memory, fMRI, febrile seizures, hippocampus, supramarginal gyrus

\section{INTRODUCTION}

It is widely believed that febrile seizures (FS) usually take benign courses and do not have adverse long-term effects on the cognitive development of children (see Hirtz, 2002 for an overview). However, there is evidence that FS are related to hippocampal injuries (e.g., Cendes, 2004) which in turn should entail an impairment of episodic memory. Although FS are the most common seizure disorder in childhood (prevalence 2-5\%; Yilmaz et al., 2008; Nakayama, 2009), memory functions have hardly been investigated so far. The goal of the present study was to identify possible FS-related modifications in the neural structures underlying episodic memory by means of functional MRI.

Febrile seizures are convulsions brought on by fever between the ages of 6 months and 5 years. One third of the affected children suffer more than one FS during infancy. FS can be divided into two groups, simple and complex. Seventy to $75 \%$ of children suffer from simple FS that are generalized and last less than $15 \mathrm{~min}$. Complex FS are either focal or prolonged (longer than $15 \mathrm{~min}$ ), or they are multiple seizures that occur within $24 \mathrm{~h}$. To allow assumptions about the consequences of FS on memory development in general we included a representative cross-section of FS characteristics in a sufficiently large sample with a mixture of simple and complex FS.

Studies have repeatedly observed hippocampal injuries among children and adults with prior FS for both the simple type (Auer et al., 2008) and the complex type (e.g., Fisher et al., 1998; Van Landingham et al., 1998; Sokol et al., 2003; Cendes, 2004). Although it remains unclear whether FS cause abnormalities in the medial temporal lobe (MTL; Falconer, 1974; Bruton, 1988; Cendes et al., 1993; French et al., 1993; Huang and Chang, 2009) or whether pre-existing brain anomalies induce FS (Hesdorffer et al., 2008), the converging evidence for hippocampal injuries being 
related to FS gives rise to the assumption that FS children should exhibit specific impairments in declarative long-term memory. Declarative memory consists of two subsystems: episodic memory (memory of events) and semantic memory (context free knowledge; Tulving, 1972). It is well established that episodic memory depends on the integrity of the hippocampus (Vargha-Khadem et al., 1997; Eichenbaum and Cohen, 2001) whereas semantic memory seems to be more dependent on perirhinal and entorhinal cortices (Vargha-Khadem et al., 1997; Gadian et al., 2000). If FS are associated with hippocampal injuries, episodic memory functions should be particularly affected.

So far, only few studies have examined episodic memory functions after FS. Animal studies that simulate FS by inducing hyperthermic seizures in young rats demonstrate that the experimentally induced seizures lead to poor episodic memory performance at a later age (Nealis et al., 1978; Chang et al., 2003). In contrast, the few existing studies investigating human subjects found impairments only for children with specific seizure characteristics. Chang et al. (2001) found reduced recognition memory performance among 6 to 9-year-old children only when FS had occurred within the first year of life. Kölfen et al. (1998) observed impairments in visualspatial memory in 6- to 9-year-old children only after complex FS. In sum, most FS during infancy do not adversely affect memory (Baram and Shinnar, 2001; Hirtz, 2002) and yet the suspicion that there is an interrelation between FS and memory modifications has not been completely dispelled.

The idea of memory modifications was fueled by new evidence in an earlier study in which we tested the memory functions of 7 to 9-year-old children with standardized neuropsychological tests and an item recognition experiment with EEG recording and in which hippocampal volumes were additionally examined (Kipp et al., 2010). In all tests, children with FS achieved the same memory performance as control children, and no significant differences in hippocampal volumes were found. However, the event-related potentials (ERP) provided evidence for modified subprocesses of recognition memory. According to dual-process theories of recognition two retrieval processes contribute to recognition: familiarity and recollection (Mecklinger, 2000; Yonelinas, 2002). The analysis of the ERP correlates of familiarity and recollection revealed deficits in recollection-based remembering in children with FS, a memory process which is assumed to rely on the integrity of the hippocampus. In contrast, familiarity-based remembering seemed to be intact. The absence of structural changes in the hippocampus together with the missing ERP correlate of recollection in the FS group led to the conclusion that FS go along with subtle functional changes in memory mechanisms mediated by the MTL. Neuropsychological tests as well as simple designed memory experiments seem to lack the required sensitivity to detect these FS-related modifications in episodic memory (Kipp et al., 2010). The current study was conducted to underpin this claim and to identify FSrelated modifications in the neural basis of recognition memory with functional MRI.

Functional imaging studies have identified activation patterns in the MTL associated with recollection and familiarity (see Eichenbaum et al., 2007 for a review). Hippocampal activation during both encoding and retrieval seems to reflect stronger recollection-based processes by associating items and their contexts and seems to be insensitive to changes in familiarity strength (e.g., Davachi et al., 2003; Ranganath et al., 2003; Daselaar et al., 2006). In contrast, the perirhinal cortex contributes to familiarity-based retrieval (Davachi et al., 2003; Wais, 2008). It is also well established that the strength of familiarity modulates lateral prefrontal and dorsal parietal activity in humans, while recollection engages primarily the ventral parietal cortex (Cabeza et al., 2008; Mecklinger, 2010).

Notably, the current knowledge on the neural correlates of recognition memory is mostly based on studies with adults. Little is known about the neural characteristics of recognition memory in children. There only exist few functional magnetic resonance imaging (fMRI) memory studies with school-aged children that all concentrate on memory encoding processes. A study by Chiu et al. (2006) showed activation in the left posterior MTL for 7 to 8 - and 10 to 18 -year-old children during story encoding predicting subsequent memory. Activation in the left anterior MTL (including the hippocampus proper) and the left prefrontal cortex was apparent only in the older child group (see also Menon et al., 2005). However, Ofen et al. (2007) found greater fMRI activation for items later remembered than for items later forgotten in PFC and MTL regions in 8 to 24 -year-old subjects. While the activation in MTL was not influenced by age, there was an age-related increase of activation in the PFC. This suggests a differential maturation of memory-relevant brain regions in the PFC and the MTL from early school age to adulthood.

To investigate changes in the neural correlates of episodic memory after FS we examined 7 to 9-year-old children who had suffered from FS in infancy and an age-matched control group. The FS group consisted of children with simple and complex FS with mainly benign courses which represents a cross-section of FS characteristics. In the face of this heterogeneity we complemented our group analyses by additional tests to control for effects of seizure type (simple vs. complex) and the number of episodes during infancy. Both child groups participated in a continuous recognition memory task (Jessen et al., 2001; Czernochowski et al., 2009) with fMRI measurement covering the entire brain. In this task, pictures were presented in random order and every picture was repeated once during the run. Participants had to indicate by button press whether a picture was presented for the first time (new item) or the second time (old item). As encoding and retrieval phases are not conducted separately, this experimental paradigm has a high ecological validity: as in real world situations it entails the simultaneous demands to detect and encode new items and to retrieve old items from memory. Also, our experience with memory studies with children revealed that old/new judgments can be easier instructed when the old/new status of items is manipulated within a single block (as in the continuous paradigm). Consistent with earlier studies (Brozinsky et al., 2005; Johnson et al., 2008; Yassa and Stark, 2008) we use this paradigm to study retrieval-related brain activation.

Based on the assumption that FS can be associated with selective injuries of the hippocampus we expected to find changes in the blood-oxygenation-level dependent (BOLD) signal within the MTL for the FS group compared to controls. Compensatory activity in the prefrontal cortex or in parietal regions was also deemed possible. 
Table 1 | Demographic data of control and FS groups.

\begin{tabular}{lll}
\hline & Control group & FS group \\
\hline$N$ & 14 & 13 \\
Male/female & $10 / 4$ & $7 / 6$ \\
Age & $8 ; 04(6 ; 11-9 ; 11)$ & $8 ; 00(7 ; 01-9 ; 11)$ \\
Age when first FS occurred & - & $2 ; 01(0 ; 08-3 ; 00)$ \\
Seizure type & - & 7 simple/6 complex \\
& & 2 focal \\
& & $4>15$ min \\
Number of episodes & - & 3 recurrent \\
& & $2.85(1-8)$ \\
\hline
\end{tabular}

\section{MATERIALS AND METHODS PARTICIPANTS}

The FS group consisted of 18 children who had been treated at Saarland University Hospital after suffering the first febrile convulsion. Five children were excluded from analysis because of movement artifacts or technical failure. Of the remaining 13 children seven had suffered from simple and six from complex FS. Seven children suffered from more than one FS during infancy, and for three of the children the first FS occurred before the age of one. None of the children was on regular medication, showed noticeable abnormalities in the EEG, or had developed epilepsy.

Eighteen control children were recruited from local elementary schools. Movement and technical artifacts made us exclude three children. One child was excluded because of a cardiac defect in the 37th week of pregnancy. The remaining 14 control children entered the statistical analysis. The two child groups were age matched (FS: 8;00 years; control: 8;04 years). All participants had normal or corrected to normal vision, and in each group 11 children were right-handed. Further details about the groups and the FS characteristics are presented in Table 1. The children's parents gave written informed consent prior to participation. The participants were paid $8 € / \mathrm{h}$ plus $12 € /$ session for travel expenses.

The current study was approved by the Ethics Committee of the Saarland Medical Association (ID No. 151/07).

\section{PROCEDURE}

The children performed two sessions. In the first session (duration $1 \mathrm{~h}$ ) we examined the general performance in different memory domains and in intellectual functioning to establish a correspondence to prior behavioral research on memory development after FS. Since the sample largely overlapped with the sample described by Kipp et al. (2010) we did not expect to find differences between the FS and control group. However, we report these results together with the fMRI results here to provide a comprehensive overview of FS-related memory modifications.

In the second session we ran a continuous recognition experiment with fMRI $(1 \mathrm{~h})$. The data of a third session in which the children performed an item recognition experiment with EEG recording is published elsewhere (Kipp et al., 2010).

\section{Neuropsychological assessment}

Intellectual functioning. To control for possible differences in intellectual functioning between the control and the FS group we used the Raven's colored progressive matrices test, a multiple choice test on abstract reasoning (Raven et al., 2002).

Working memory. We administered the forward and backward digit span test of the HAWIK-R (German version of the WISC; Tewes, 1997).

Semantic memory. Three additional subtests of the HAWIK-R served to assess semantic memory: general knowledge measures factual knowledge, general comprehension measures knowledge about standard behavior patterns, and vocabulary measures word knowledge.

Episodic memory. To test verbal episodic memory the Verbaler Lern- und Merkfähigkeitstest (VLMT; Schweisthal, 1997; Helmstaedter et al., 2001) was used [German version of the auditory verbal learning test (AVLT)]. In this test children have to learn a list of 15 words and are assessed with respect to immediate and delayed recall, recognition, learning gradient, and interference sensitivity. To assess performance in visual episodic memory we used the Rey-Osterrieth complex figure (Osterrieth, 1944). In this test children have to copy an abstract line drawing, and to reproduce it from memory immediately afterward and again after a delay of $30 \mathrm{~min}$.

Differences in the neuropsychological test performance between the control and the FS group were assessed by means of two-tailed $t$-tests. Additional analyses served to see whether the following seizure characteristics within the FS group differentially affected performance: seizure type (simple vs. complex) and number of episodes during infancy (one vs. more). According to the small subgroups (seven simple vs. six complex, six one vs. seven more) we used Mann-Whitney $U$ tests. Since, in the current sample, the two subgroups of children having had an onset of the first FS within the first year of life $(N=3)$ and after $(N=10$; see Chang et al., 2001) were quite different in size we refrained from analyzing this additional FS characterization.

\section{Continuous recognition experiment with fMRI}

Stimuli. The stimuli were 90 colored line drawings of everyday objects from the Snodgrass and Vanderwart catalog (Rossion and Pourtois, 2004). Each picture was framed within an area of $280 \times 280$ pixels.

Procedure. The continuous recognition experiment consisted of three blocks. In each block, 20 pictures were presented consecutively. Each picture was repeated once during the run with a lag of $10-15$ intervening items. Additional 10 pictures were used as filler items to enable the lag manipulations for the targets. To receive the same proportion of old and new items these filler items were also repeated with differing lags but they did not enter statistical analyses ${ }^{1}$. This resulted in a total of 60 trials per block. Participants had to indicate by button press whether the displayed picture was presented for the first ("new") or the second time ("old"). Each

\footnotetext{
${ }^{1}$ We performed additional analyses that included targets plus filler items. The results correspond to the analyses without filler items. Since for the targets the repetition lag was precisely controlled we report the analyses based on targets only.
} 
trial started with a blank screen $(250 \mathrm{~ms})$ followed by a fixation cross $(250 \mathrm{~ms})$ and the presentation of a picture $(1000 \mathrm{~ms})$. The variable inter-stimulus interval was normal distributed with a mean of $4500 \mathrm{~ms}$ (range: $2000-7000 \mathrm{~ms}$ ). The trial sequence was pseudo-randomized such that no more than four old or four new stimuli succeeded each other. After each block feedback for accuracy was given and the next block started immediately. Prior to the experiment, a short practice block was performed outside the scanner.

Behavioral analyses. Memory accuracy was analyzed by means of the proportion of correct old (HITs) and new responses [correct rejections (CRs)] and the discrimination index Pr, which is calculated by subtracting the proportion of false alarms to new items (FA) from the proportion of HITs (Snodgrass and Corwin, 1988). Response bias (Br) was defined as FA/(1-Pr), assessing the willingness of saying "old" when in an uncertain state (Snodgrass and Corwin, 1988). Differences between control and FS group with respect to memory accuracy measures and response times were assessed by means of two-tailed $t$-tests for independent samples. Since the two child groups differed with respect to gender distribution we conducted additional ANCOVAs with gender as a covariate. The results perfectly correspond with the results of the $t$-tests. Thus, only results of the $t$-tests are reported below. In addition, we used Mann-Whitney $U$ tests to test whether different seizures characteristics (seizure type and number of episodes during infancy) affected performance in a different way.

MRI data acquisition. Structural and functional brain imaging was performed on a $1.5 \mathrm{~T}$ Siemens Sonata scanner. A T1 weighted 3D whole-brain scan was performed for anatomical coregistration (MP-Rage sequence: $\mathrm{TR}=1900 \mathrm{~ms}, \mathrm{TE}=4 \mathrm{~ms}$, flip angle $=15^{\circ}, \mathrm{FOV}=256 \mathrm{~mm}, 176$ sagittal slices). During functional imaging, 20 axial slices ( $4 \mathrm{~mm}$ thickness, $1 \mathrm{~mm}$ inter-slice distance, $\mathrm{FOV}=224 \mathrm{~mm}, 64 \times 64$ data acquisition matrix) were acquired with a T2* weighted BOLD sensitive gradient echo planar sequence $(\mathrm{TR}=1800 \mathrm{~ms}, \mathrm{TE}=50 \mathrm{~ms}$, inter-slice time $90 \mathrm{~ms}$, flip angle $=85^{\circ}$ ). A total of 605 functional volumes were taken during each experimental run.

fMRI data analyses. Analyses of fMRI data were carried out using the BrainVoyager software package (Brain Innovation B.V., Maastricht, The Netherlands). Due to excessive movement artifacts in the last third of the experiment in most children $(z$ translation $\geq 6 \mathrm{~mm}$ ), only the first and second block of the experiment (i.e., 396 volumes) entered the preprocessing and statistical analyses. The four initial volumes were excluded from further processing due to their stronger $\mathrm{T} 1$ saturation. The remaining volumes were preprocessed using the standard routines as implemented in BrainVoyager. First, to correct for the sequentially executed interleaved slice acquisition a slice scan time correction was performed using sinc interpolation. Next, a correction of 3D motion (sinc interpolation) was performed to spatially align all functional volumes (of both blocks) to the first acquired volume. An isotropic spatial Gaussian filter (FWHM $=6 \mathrm{~mm}$ ) was then applied to the data. To ensure that differences between the mean signals between the two blocks do not mask the smaller differences of interest between the conditions the time course across blocks was normalized using a $z$-transformation procedure. After linear trend removal a temporal high pass filter with a cut-off frequency of two cycles (equalling $1 / 40 \mathrm{~Hz}$ ) was applied to account for low frequency signal changes and baseline drifts. Subsequent statistical analyses were adjusted for increased autocorrelations caused by filtering. Functional slices were then co-registered to the high-resolution whole-brain anatomical scans obtained in the beginning of the sessions, and were subsequently spatially transformed into stereotactic Talairach space (Ofen et al., 2007) and re-sampled to a spatial resolution of $3 \mathrm{~mm} \times 3 \mathrm{~mm} \times 3 \mathrm{~mm}$.

The hemodynamic response function (HRF) was computed as two gamma functions (onset: 0 , time to response peak: $5 \mathrm{~s}$, time to undershoot peak: $15 \mathrm{~s}$ ). The design matrix for each participant included four predictors reflecting the participants' item-specific HRFs and a predictor reflecting the signal quality computed during the mean intensity adjustment procedure. In addition, motion parameters were added as predictors of no interest to the design matrix of each block. The four item status specific predictors modeled the correct rejection of the first presentation of a picture ("CR"), the correct recognition of its second occurrence ("HIT"), false-positive responses to the first occurrence of an item ("FA"), and the miss of the repetition of an object ("Miss").

In a first step, statistical analyses of the resulting percent signal change values for correct responses (HIT vs. CR) were carried out for all subjects collapsed across groups using the multistudy GLM random effects analysis tool as implemented in the BrainVoyager software package. The GLM design comprised the within-subject factor item status (HIT vs. CR). In a second step, between-group comparisons were analyzed with a GLM design including the between-subject factor group (control vs. FS) and the within-subject factor item status (HIT vs. CR). Since the numbers of FAs and Misses were small these item categories were not analyzed. To restrict analyses to the most important activations, only results exceeding a significance level of $p=0.005$, also used in other studies (e.g., Ment et al., 2006), with a 25 voxel extent threshold (cluster level corrected at $p<0.05$ ) are reported here. Regions of interest (ROI) were defined based on clusters of activity in this second analysis. The mean percent signal change of these ROIs was subjected to further analyses to estimate the BOLD response to HITs and CRs separately for the control and FS group.

\section{RESULTS NEUROPSYCHOLOGICAL ASSESSMENT}

The results of the control and FS group in the neuropsychological tests are presented in Table 2. The two groups did not differ in any of the tests: there were no differences in intellectual functioning (colored progressive matrices), semantic memory (general knowledge, general comprehension, vocabulary), and verbal (VLMT) or visual episodic memory (Rey-Osterrieth complex figure). Only the performance in the working memory task (digit span) differed marginally between-groups $(p=0.07)$, the FS group outperforming the control group. Considering all test results, the numerical differences between-groups had different directions, e.g., in the semantic memory tests the control group was slightly better than the FS group in general knowledge but performed slightly worse in general comprehension and 
Table 2 | Neuropsychological assessment: results for the control and FS groups (SE in parentheses).

\begin{tabular}{|c|c|c|c|}
\hline Cognitive ability & Control group & FS group & $\begin{array}{l}p \text { Values } \\
\text { (t-tests) }\end{array}$ \\
\hline \multicolumn{4}{|c|}{ INTELLECTUAL FUNCTIONING } \\
\hline Colored progressive matrices & $29.59(1.26)$ & $30.08(0.98)$ & 0.46 \\
\hline \multicolumn{4}{|l|}{ WORKING MEMORY } \\
\hline Digit span (HAWIK-R)* & $10.92(0.33)$ & $12.92(0.71)$ & 0.07 \\
\hline \multicolumn{4}{|c|}{ SEMANTIC MEMORY (HAWIK-R)* } \\
\hline General knowledge & $12.79(0.48)$ & $11.62(0.59)$ & 0.24 \\
\hline General comprehension & $12.79(0.69)$ & $13.23(0.43)$ & 0.64 \\
\hline Vocabulary & $13.79(0.53)$ & $14.31(0.62)$ & 0.60 \\
\hline \multicolumn{4}{|l|}{ EPISODIC MEMORY } \\
\hline \multicolumn{4}{|l|}{ Verbal memory $(\mathrm{VLMT})^{* *}$} \\
\hline Immediate recall & $7.36(0.41)$ & $6.62(0.38)$ & 0.29 \\
\hline Learning gains & $49.21(2.35)$ & $46.46(2.64)$ & 0.55 \\
\hline Recall after interference & $10.07(0.74)$ & $9.77(0.53)$ & 0.79 \\
\hline Delayed recall & $10.43(0.65)$ & $10.46(0.63)$ & 0.98 \\
\hline Loss after delay & $1.50(0.44)$ & $0.92(0.45)$ & 0.48 \\
\hline Recognition & $12.64(0.92)$ & $13.67(0.32)$ & 0.50 \\
\hline \multicolumn{4}{|c|}{ Visual memory (rey-osterrieth complex figure) } \\
\hline Copy & $28.71(1.22)$ & $30.50(0.96)$ & 0.37 \\
\hline Immediate recall & $15.54(1.39)$ & $16.54(1.37)$ & 0.69 \\
\hline Delayed recall & $16.12(1.35)$ & $14.42(1.19)$ & 0.47 \\
\hline
\end{tabular}

All scores except for HAWIK-R scores are raw scores.

${ }^{*} H A W I K-R$ (Tewes, 1997) is the German version of the WISC. Scores are standardized scores based on chronological age norms (mean $=10, S D=3$ ).

${ }^{* *}$ VLMT (Helmstaedter et al., 2001) is the German version of the auditory verbal learning test (AVLT).

vocabulary. Therefore, we assume that there are no systematic group differences.

Additional tests for the FS group did not reveal any relations between test results and FS characteristics, i.e., seizure type (simple vs. complex) and number of episodes during infancy (one or more).

\section{CONTINUOUS RECOGNITION EXPERIMENT WITH FMRI Behavioral results}

To mirror the analyses of behavioral and fMRI data, only the trials from the first and second block of the continuous recognition memory task were analyzed (see Table 3 ). In both child groups the number of trials with missing responses was equal [control group: 1.71; FS group: $1.31 ; t(25)=0.51, p=0.62$ ]

There were no significant differences in memory accuracy between the two child groups. Both groups reached the same proportion of HITs $[t(25)=1.68, p<0.11]$, the same proportion of CRs $[t(25)=0.07, p=0.95]$ and a similar Pr score $[t(25)=0.86$, $p=0.40]^{2}$. There was also no difference in response bias $\mathrm{Br}$ $[t(25)=1.35, p=0.19]$, indicating a rather neutral response bias in both groups with a numerically more conservative bias in the FS group.

${ }^{2}$ A second set of analyses based on absolute numbers of HITs and CRs (raw scores) as dependent variables yielded statistically identical results.
Table 3 | Recognition memory performance (SE of the means are given in parentheses).

\begin{tabular}{lcc}
\hline & \multicolumn{1}{c}{ Control group } & FS group \\
\hline \multicolumn{2}{l}{ MEAN NUMBER OF RESPONSES } & \\
HIT & $33.43(1.06)$ & $31.15(1.22)$ \\
CR & $32.93(1.76)$ & $32.77(0.86)$ \\
PROPORTION OF RESPONSES & \\
HIT & $0.85(0.03)$ & $0.78(0.03)$ \\
CR & $0.84(0.04)$ & $0.84(0.02)$ \\
PR & $0.69(0.06)$ & $0.63(0.05)$ \\
Br & $0.49(0.03)$ & $0.43(0.03)$ \\
MEAN REACTIONTIME (ms) & \\
HIT & $999(51)$ & $984(78)$ \\
CR & $990(41)$ & $966(66)$
\end{tabular}

Regarding reaction times there were again no differences between the two child groups neither for HITs $[t(25)=0.16$, $p=0.87]$ nor for CRs $[t(25)=0.31, p=0.76]$.

Using Mann-Whitney $U$ tests we explored whether the following FS characteristics affected memory accuracy and reaction times differently: seizure type (simple vs. complex; all $p$ values $>0.43$ ) and number of episodes during infancy (one vs. more FS; all $p$ values $>0.15$ ). In sum, the control and the FS group did not differ in terms of memory accuracy, response bias and reaction times.

\section{fMRI results}

Table 4 gives an overview of all fMRI results reported in the following. In a first analysis we included all children irrespective of their group membership and contrasted the activation of HITs and CRs. HITs led to decreased activity in two brain voxel clusters along the superior temporal sulcus (STS, BA 22). In both significant STS clusters more than half of the voxels are located in the middle temporal gyrus (MTG) and the remaining voxels in the superior temporal gyrus (STG). In contrast, HITs gave rise to increased activity in several brain areas, namely the right middle frontal gyrus (BA 6/10), left thalamus, left parahippocampal gyrus (BA 36), and left precentral gyrus (BA 4). No differences in brain activity between HITs and CRs were observed in bilateral hippocampus. The results are illustrated in Figure 1.

The second analysis was conducted to evaluate possible differences between the two child groups. A statistically significant between-group difference with respect to the activation in HITs and CRs was found in the right supramarginal gyrus within the inferior parietal lobule (BA 40, 48-51, 27, cluster size: 27 voxels). This finding was confirmed statistically by a ROI analysis $(2 \times 2$ ANOVA with the factors group and item status) performed for all activated voxels in the inferior parietal lobe. The interaction between both factors was highly significant $[F(1,25)=15.59$, $p<0.001]$. Post hoc tests indicated that in the control group CRs showed greater activation than HITs (Scheffé-test, $p<0.01$ ), whereas in the FS group CRs and HITs did not differ with respect to activation $(p=0.38)$. This result did not change when Gender was included as a covariate. The interaction pattern is illustrated in Figure 2. 
Table 4 | Brain regions showing significant differences in BOLD signals for HITs and CRs $(p<005)$.

\begin{tabular}{|c|c|c|c|c|c|c|c|c|}
\hline & \multirow[t]{2}{*}{ Brain region } & \multirow[t]{2}{*}{$\mathbf{B A}^{*}$} & \multirow[t]{2}{*}{ Left/right } & \multirow[t]{2}{*}{ No. of voxels } & \multirow[t]{2}{*}{$t$ Value } & \multicolumn{3}{|c|}{ Peak Talairach coordinates } \\
\hline & & & & & & $\boldsymbol{x}$ & $y$ & $z$ \\
\hline \multirow[t]{2}{*}{ Main effect: HIT < CR } & Superior temporal sulcus & 22 & $\mathrm{R}$ & 39 & -3.59 & 61 & -29 & 3 \\
\hline & & 22 & $\mathrm{R}$ & 215 & -3.63 & 57 & -41 & 6 \\
\hline \multirow[t]{5}{*}{ Main effect: HIT > CR } & Middle frontal gyrus & 6 & $\mathrm{R}$ & 431 & 4.40 & 26 & -6 & 38 \\
\hline & & 10 & $\mathrm{R}$ & 100 & 3.64 & 30 & 33 & 11 \\
\hline & Thalamus (lateral posterior nucleus) & & $\mathrm{L}$ & 75 & 3.70 & -22 & -20 & 18 \\
\hline & Parahippocampal gyrus & 36 & $\mathrm{~L}$ & 29 & 3.62 & -29 & -40 & -6 \\
\hline & Precentral gyrus & 4 & $\mathrm{~L}$ & 85 & 3.62 & -48 & -6 & 42 \\
\hline Interaction: group $\times$ item status & Supramarginal gyrus & 40 & $\mathrm{R}$ & 27 & & 48 & -51 & 27 \\
\hline
\end{tabular}

${ }^{*}$ According to Talairach and Tournoux (1988).

The influence of different FS characteristics on the difference in the BOLD signal of HITs and CRs within the right supramarginal gyrus (BA 40) was explored with additional analyses for the FS group only. Mann-Whitney $U$ tests could show that neither seizure type $(p=0.78)$ nor number of episodes during infancy $(p=0.48)$ affected the difference in BOLD signal between HITs and CRs.

\section{DISCUSSION}

To our knowledge, this is the first study to examine declarative memory processes in school-aged children who had suffered from FS in early childhood using an event-related fMRI design. Although we did not find differences in memory accuracy between control and FS group on the behavioral level the fMRI analyses revealed highly selective changes in the hemodynamic activity.

With standardized neuropsychological tests we examined performance in different cognitive domains: intellectual functioning, working memory, semantic memory, and episodic memory. In all tests the FS group reached the same level of performance as the control group. The same holds for memory accuracy and response speed in the continuous recognition memory experiment in which the two child groups did not differ. Furthermore, memory performance in FS children was not negatively influenced by the complexity of FS, or the number of episodes during infancy. We are aware of the fact that the current sample is quite small. However, the results of overlapping and enlarged groups [ 17 children/group; reported by Kipp et al. (2010)] did not reveal any FS-related group differences in the neuropsychological tests too. Against the background of a possible functional hippocampal damage related to FS one could have expected lower performance selectively in episodic memory tests. However, most studies indicate that FS do not have adverse long-term effects on cognition (for an overview see Hirtz, 2002) and a minority of studies provide evidence for reduced episodic memory performance only for subgroups, e.g., for children with complex FS (Kölfen et al., 1998). Since we did not even find lower memory performance for children with complex FS or children who suffered from more than one FS during infancy we assume that FS-related memory deficits are too subtle and specific to be detected by neuropsychological tests (Kipp et al., 2010). In addition, the current continuous recognition memory experiment in which the repetition lack did not exceed $90 \mathrm{~s}$, may have been too easy to reveal FS-related impairments in episodic memory on the behavioral level.

Only a few studies have explicitly examined episodic memory in children by means of fMRI recordings. Therefore, in the following we refer to adult studies when discussing the activation pattern of the control and the FS group. It must be recognized that in principal the HRFs could be coupled differently in children and adults. But at least in some brain areas including the MTL region only minimal differences in the time courses and locations of functional activation foci do appear between children and adults (Kang et al., 2003; Ofen et al., 2007).

The fMRI analysis of our continuous recognition experiment revealed several effects of item status (HITs vs. CRs) that were independent of group membership. First, repetition suppression effects (i.e., lower BOLD signal for HITs than for CRs) were visible in the right STS. This is an untypical region for recognition memory tasks. However, the MTG and, to a lesser degree, the STG have been associated with semantic priming, i.e., reduced activation for semantically primed than unprimed events (for an overview see Lau et al., 2008). Since in the observed significant STS cluster of our study more than half of the voxels lie within the MTG, and the remaining voxels within the STG it is possible that the observed pattern indicates a priming effect in the sense that for repeatedly presented items (HITs) the access to stored conceptual representations is facilitated and therefore the BOLD activity is reduced. So far, semantic priming effects within the MTG and STG have been demonstrated with lexical material only. Studies usually devised tasks which required the semantic categorization of words or judgments on the words' semantic properties (see Lau et al., 2008). Similar processes may have been at work in our present task, even though pictorial materials were used.

Second, we observed repetition enhancement effects in both child groups (i.e., higher BOLD signal for HITs than for CRs) in prefrontal brain regions, the thalamus, and parahippocampal gyrus. Such repetition enhancement effects have already been reported in adult memory studies (Jessen et al., 2001; Henson et al., 2005; Vilberg and Rugg, 2007). The middle frontal gyrus (BA 6/10) is assumed to be one of the brain areas that are selectively sensitive to familiarity, although typically with greater involvement of the left hemisphere. For example Yonelinas et al. (2005) showed a positive correlation between BA 6/10 activity and the confidence with 

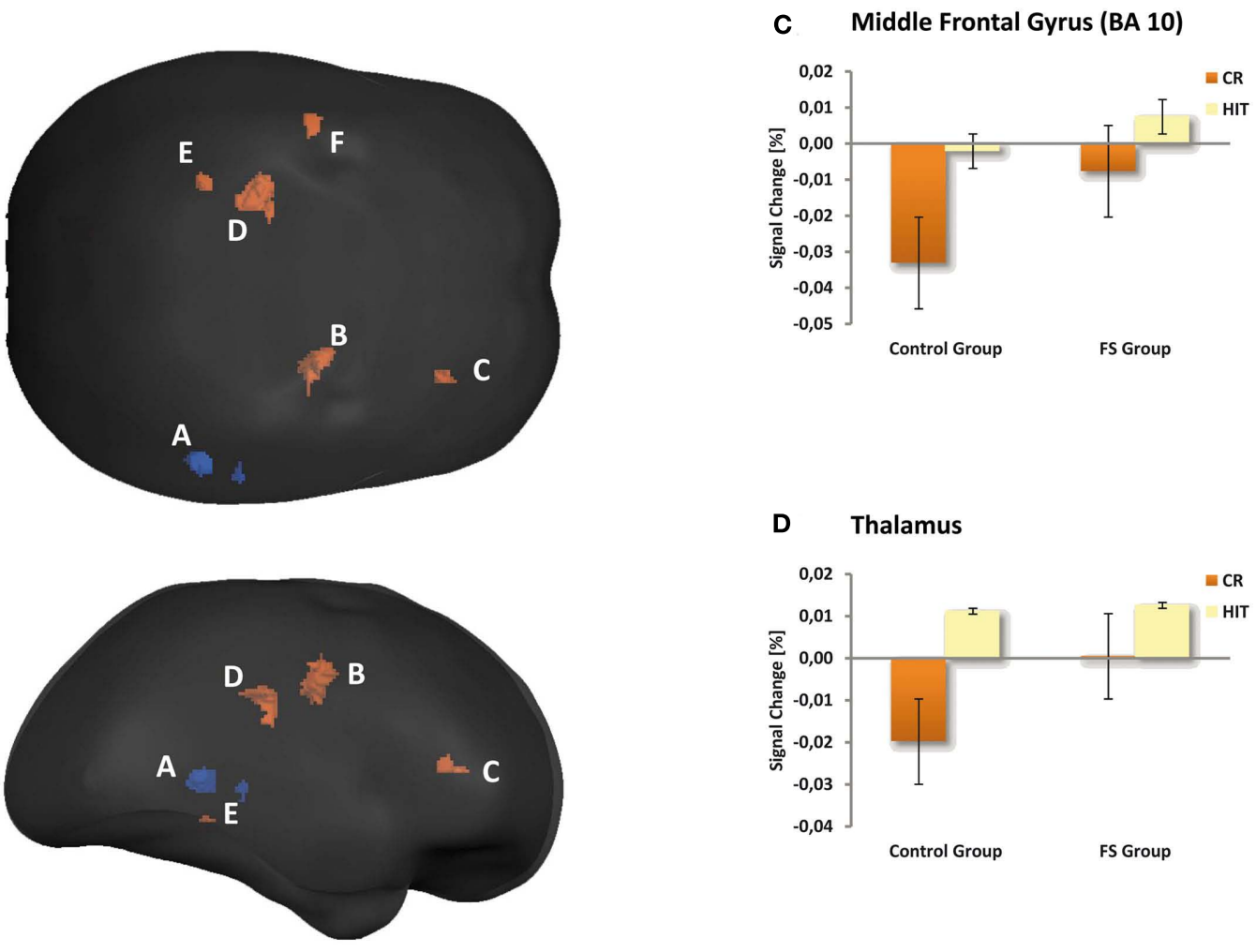

D Thalamus

A Superior Temporal Gyrus

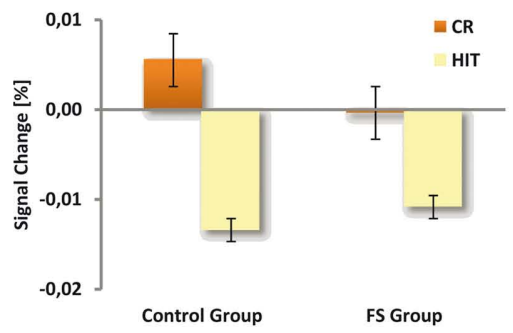

B Middle Frontal Gyrus (BA 6)

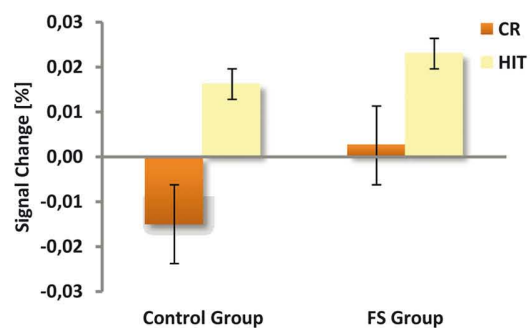

FIGURE 1 | Maps illustrating item status effects for both child groups averaged and amplitude responses plotted for the control and FS group separately (bar graphs A-F). The voxel clusters were overlaid on a

normalized individual brain. Clusters are viewed from posterior and lateral

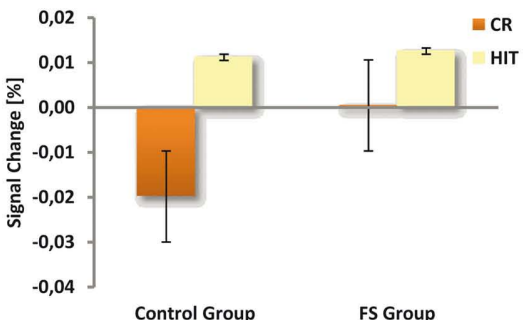

E Parahippocampal Gyrus (BA 36)

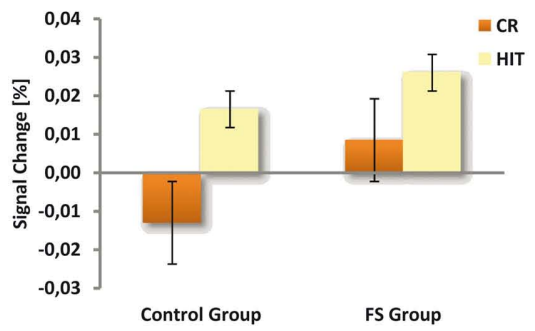

F Precentral Gyrus (BA 4)

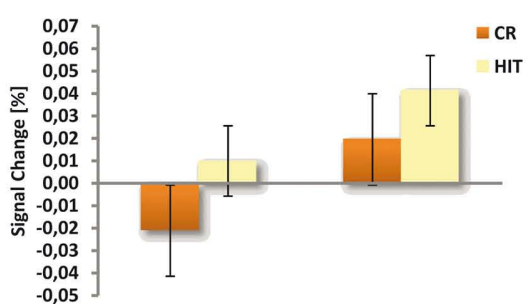

Control Group $\quad$ FS Group aspects. Blue depicts repetition suppression effects (HIT $<\mathrm{CR}$ ), red repetition enhancement effects (HIT > CR). The bar graphs illustrate the mean activity of the respective clusters. Crucially, the activation pattern in the depicted brain regions was similar in the two child groups. 

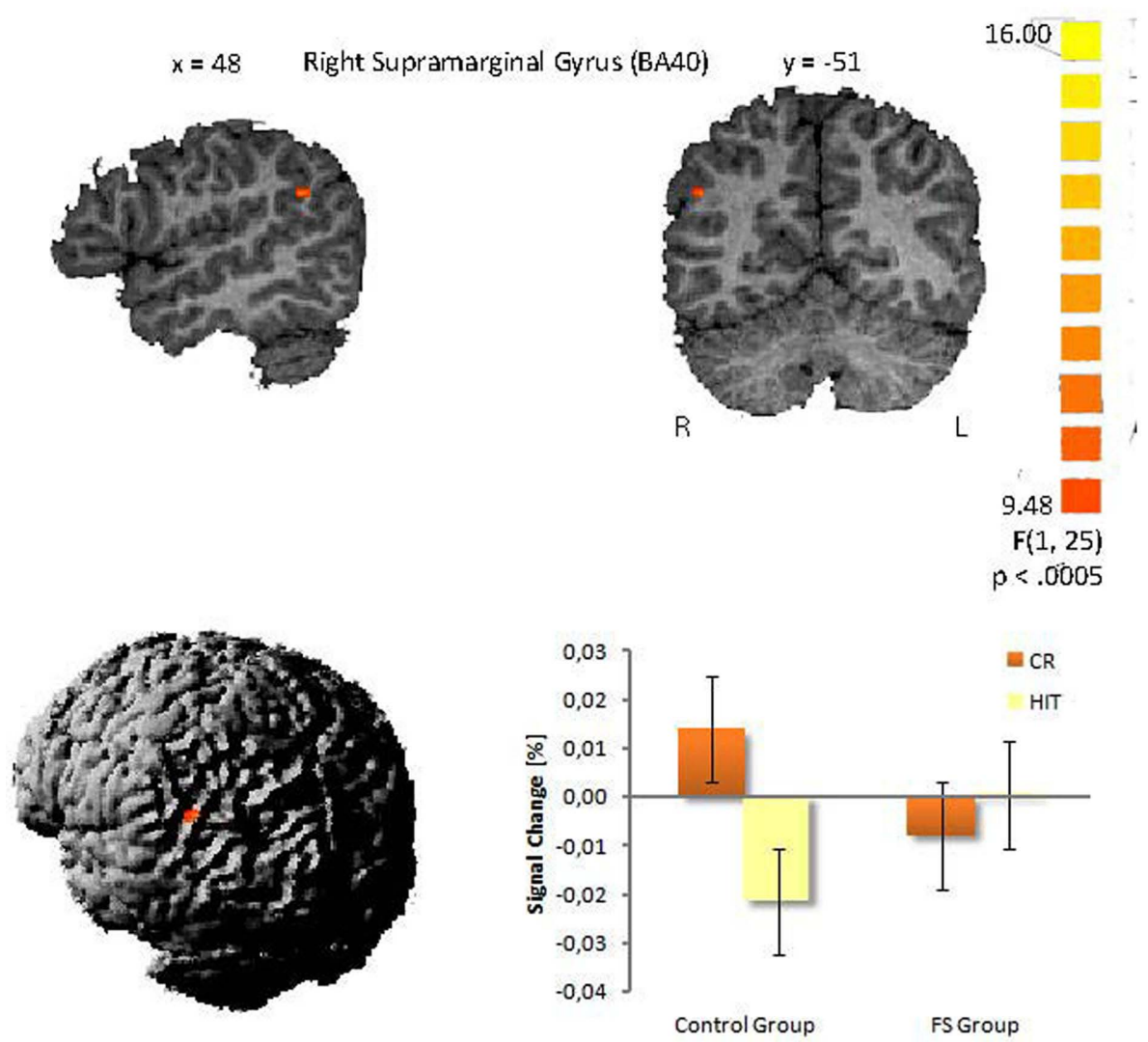

FIGURE 2 | Interaction in the right supramarginal gyrus between group and item status $(\boldsymbol{p}<\mathbf{0 . 0 0 5})$. (Talairach coordinates: $x=48, y=-51, z=27)$. The voxel clusters were overlaid on a normalized individual brain.

which familiarity-based recognition judgments were made. In line with these findings, Vilberg and Rugg (2007) using a remember/know procedure found familiarity sensitive regions in BA 6 and BA 10. For the current study this could indicate that the children judged repeated items as being old primarily on the basis of familiarity.

We did not find any significant activation clusters in the hippocampus proper. This is in line with a study by Chiu et al. (2006) who found hippocampal activation only during encoding but not during retrieval and this only for children who were 10 years or older. However, we found a repetition enhancement effect in the left posterior parahippocampal gyrus (PHG, BA 36), a region for which Daselaar et al. (2006) found a linear activation increase with increasing levels of oldness, as revealed by recognition confidence ratings. On the basis of this finding they concluded that this activity within the posterior PHG may reflect stimulus familiarity. Preston et al. (2004) also found greater activation in a region that falls into BA 36 in forced choice recognition memory judgments on learned item pairs when the single items had been seen more frequently during encoding. The authors also relate this effect in the PHG to enhanced item familiarity. This interpretation is compatible with studies showing associations between activity in this region and item-based perceptual retrieval processes (Cabeza et al., 2001; Goh et al., 2004). For the current study this could again mean that in case of repeated items the children's old judgments were primarily based on familiarity. This interpretation, however, has to be taken with caution as other studies relate activity in the posterior PHG to recollection (e.g., Eldridge et al., 2000; Cansino et al., 2002; Yonelinas et al., 2005; Ofen et al., 2007). At the present state it remains unclear whether some parts of the PHG contribute to familiarity and other parts contribute to recollection.

The primary goal of the present study was to identify modifications in the neural structures of recognition memory in school children that go along with FS. The only activation difference we could find between the control and FS group was in the right supramarginal gyrus (BA 40). The control group showed higher activity for CRs compared to HITs whereas the FS group did not show a difference between both response types.

There are two possible explanations for this interaction. First, the supramarginal gyrus seems to be associated with decision confidence in memory retrieval, i.e., items that are recognized with high confidence elicit more activity than those recognized with low confidence (Daselaar et al., 2006; Ciaramelli et al., 2008). Within this framework the differential activation patterns in the supramarginal gyrus in the two child groups could reflect differences in decision confidences for old and new items. The control group could have correctly rejected new items with higher confidence than correctly accepted old items what could have led to 
higher supramarginal activity for new items than for old items. In contrast, the FS group could have answered with the same confidence level to both categories resulting in equal activation levels in the supramarginal gyrus. The response bias analysis tentatively supports this view. Although not significant, the control children showed a more liberal response criterion, than the FS group, i.e., in case of uncertainty they more likely pressed the old button. From this it follows, that the control group gave new responses with higher certainty/confidence and this could have resulted in the enhanced supramarginal gyrus activation to correct new responses in this group. Tentative support for this view comes from marginal significant correlation between the response bias (across both groups) and the size of the activation difference between CRs and HITs in the supramarginal gyrus $(r=0.34$, $p=0.09$ ). This means the more liberal the response criterion and as a consequence of this, the higher the confidence in rejecting new items, the larger the activation for CRs compared to HITs tended to be. This interpretation, although plausible, is still speculative and needs further exploration and empirical evidence.

There is a second interpretation for the group differences within the right supramarginal gyrus. Such right lateralized activations are often reported in studies examining attentional processes in episodic memory tasks (see Hutchinson et al., 2009 for an overview). According to Quamme et al. (2010) the right supramarginal gyrus (BA 40) plays a crucial role during episodic retrieval, by allocating attention toward recollected information. This is in line with other researchers who have argued that subjects can strategically decide to rely on recollection (Hintzman and Curran, 1994; Gruppuso et al., 1997). In their fMRI study Quamme et al. (2010) found increased activation in the right supramarginal gyrus (BA 40) being associated with a selective decrease in FAs to related lures. They concluded that enhanced top-down guided attention toward recollection can prevent FAs. The precise underlying mechanism remains unclear. Quamme et al. (2010) propose two possibilities: (1) internally directed attention could directly impact the hippocampus and increase the likelihood that the hippocampus generates a recollective signal, or (2) internally directed attention could stimulate brain regions that process hippocampal signals and therefore enhance the likelihood that a recollective signal generated by the hippocampus is detected. According to these assumptions our results could be interpreted in the following manner. The control children possibly directed more attention toward recollection when they were confronted with new items in order to prevent FAs and to correctly reject new items (recall-toreject; Clark, 1992). This is plausible if one assumes that during the recognition task the children discovered semantic categories (e.g., "animals") and assigned most stimuli to a category. In our study, the pictorial stimuli were not explicitly organized along semantic

\section{REFERENCES}

Auer, T., Barsi, P., Bone, B., Angyalosi, A., Aradi, M., Szalay, C., Horvath, R. A., Kovacs, N., Kotek, G., Fogarasi, A., Komoly, S., Janszky, I., Schwarcz, A., and Janszky, J. (2008). History of simple febrile seizures is associated with hippocampal abnormalities in adults. Epilepsia 49, 1562-1569.
Baram, T., and Shinnar, S. (2001). Do febrile seizures improve memory? Neurology 57, 7-8.

Brozinsky, C., Yonelinas, A., Kroll, N., and Ranganath, C. (2005). Lag-sensitive repetition suppression effects in the anterior parahippocampal gyrus. Hippocampus 15, 557-561. categories, however, it was principally possible to group a large proportion of the stimuli according to such categories, e.g., the 90 stimuli included 20 pictures of animals, 16 of food, 9 of vehicles. When confronted with a new item that fit into a category the children could have recollected old items from this category to guide the rejection of the semantically related new item (see von Zerssen et al., 2001). For the correct recognition of old items the control children in our study did not have to rely on recollection and could have judged the item status based on item familiarity. The direction of attention toward recollection in order to correctly reject new items could have resulted in a greater stimulus-evoked BOLD response to CRs than to HITs in the control group. In contrast, children with FS may have not adopted such a strategic use of recollection and therefore did not show different BOLD signals for CRs and HITs in the supramarginal gyrus. This interpretation is consistent with our ERP study that was run with the same children (Kipp et al., 2010), in which the ERP correlate of recollection - the late parietal old/new effect - was selectively diminished in the FS group. Thus, possibly both studies indicate a highly selective deficit in recollection related processes related to FS.

\section{CONCLUSION}

In summary, the current fMRI study revealed highly selective differences in the neural activation pattern between control and FS children in the right supramarginal gyrus (BA 40) while task performance was equal. Control children showed larger stimulusevoked BOLD responses to CRs compared to HITs whereas the FS group showed similar activity for both stimulus classes. This may indicate that the control children used strategic recollection in order to reject new items (recall-to-reject) and gave old answers on basis of familiarity. In contrast, children with FS may have not directed attention toward recollection to the same degree in any stimulus class and therefore did not show different BOLD signals for CRs and HITs. This missing differentiation could be a consequence of impaired recollective processing in children with FS.

The observed modification in memory structures is especially striking given that the FS children in the study mainly had benign courses of FS. Since the memory task used in this study could be solved solely on basis of familiarity, further research is needed to find out whether FS children suffer from memory deficits on a behavioral level when the task sets high demands on recollection.

\section{ACKNOWLEDGMENTS}

This work was supported by Grant KI 1399/1-1 of the German Research Foundation (Dr. Kerstin Kipp). We thank Maria Bunge, Katrin Hegewald, Julia Hoffmann-Selzner, and Ellen Meierotto for their valuable support during data collection.
Bruton, C. J. (1988). The Neuropathology of Temporal Lobe Epilepsy. New York: Oxford University Press.

Cabeza, R., Ciaramelli, E., Olson, I. R., and Moscovitch, M. (2008). The parietal cortex and episodic memory: an attentional account. Nat. Rev. Neurosci. 9, 613-625.
Cabeza, R., Rao, S., Wagner, A., Mayer, A., and Schacter, D. (2001). Can medial temporal lobe regions distinguish true from false? An event-related functional MRI study of veridical and illusory recognition memory. Proc. Natl. Acad. Sci. U.S.A. 98, 4805-4810. 
Cansino, S., Maquet, P., Dolan, R., and Rugg, M. (2002). Brain activity underlying encoding and retrieval of source memory. Cereb. Cortex 12, 1048-1056.

Cendes, F. (2004). Febrile seizures and mesial temporal sclerosis. Curr. Opin. Neurol. 17, 161-164.

Cendes, F., Anderman, F., Dubeau, F., Gloor, P., Evans, A., JonesGotman, M., Olivier, A., Andermann, E., Tobitaille, Y., and LopesCendes, I. (1993). Early childhood prolonged febrile convulsions, atrophy and sclerosis of mesial structures, and temporal lobe epilepsy: an mri volumetric study. Neurology 43, 1083-1087.

Chang, Y., Huang, A., Kuo, Y., Wang, S., Chang, Y., and Huang, C. (2003). Febrile seizures impair memory and cAMP response-element binding protein activation. Ann. Neurol. 54, 706-718.

Chang, Y. C., Guo, N. W., Wang, S. T., Huang, C. C., and Tsai, J. J. (2001). Working memory of schoolaged children with a history of febrile convulsions: a population study. Neurology 57, 37-42.

Chiu, C.-Y., Schmithorst, V. J., Brown, R. D., Holland, S. K., and Dunn, S. (2006). Making memories: a crosssectional investigation of episodic memory encoding in childhood using fMRI. Dev. Neuropsychol. 29, 321-340.

Ciaramelli, E., Grady, C., and Moscovitch, M. (2008). Top-down and bottom-up attention to memory: a hypothesis (AtoM) on the role of the posterior parietal cortex in memory retrieval. Neuropsychologia 46, 1828-1851.

Clark, S. E. (1992). Word frequency effects in associative and item recognition. Mem. Cognit. 20, 231-243.

Czernochowski, D., Mecklinger, A., and Johansson, M. (2009). Age-related changes in the control of episodic retrieval: an ERP study of recognition memory in children and adults. Dev. Sci. 12, 1026-1040.

Daselaar, S. M., Fleck, M. S., and Cabeza, R. (2006). Triple dissociation in the medial temporal lobes: recollection, familiarity and novelty. J. Neurophysiol. 96, 1902-1911.

Davachi, L., Mitchell, J. P., and Wagner, A. D. (2003). Multiple routes to memory: distinct medial temporal lobe processes build item and source memories. Proc. Natl. Acad. Sci. U.S.A. 100, 2157-2162.

Eichenbaum, H., and Cohen, N. J. (2001). From Conditioning to Conscious Recollection: Memory Systems of the Brain. New York: Oxford University Press.

Eichenbaum, H., Yonelinas, A., and Ranganath, C. (2007). The medial temporal lobe and recognition memory. Annu. Rev. Neurosci. 30, 123-152.

Eldridge, L., Knowlton, B., Furmanski, C., Bookheimer, S., and Engel, S. (2000). Remembering episodes: a selective role for the hippocampus during retrieval. Nat. Neurosci. 3, 1149-1152.

Falconer, M. A. (1974). Mesial temporal (Ammon's horn) sclerosis as a common cause of epilepsy: aetiology, treatment, and prevention. Lancet 2, 767-770.

Fisher, P. D., Sperber, E. F., and Moshe, S. L. (1998). Hippocampal sclerosis revisited. Brain Dev. 20, 563-573.

French, J. A., Williamson, P. D., Thadani, V. M., Darcey, T. M., Mattson, R. H., Spencer, S. S., and Spencer, D. D. (1993). Characteristics of medial temporal lobe epilepsy: I. Results of history and physical examination. Ann. Neurol. 34, 774-780.

Gadian, D. G., Aicardi, J., Watkins, K. E., Porter, D. A., Mishkin, M., and Vargha-Khadem, F. (2000). Developmental amnesia associated with early hypoxic-ischaemic injury. Brain 123, 499-507.

Goh, J. S., Siong, S., Park, D., Gutchess, A., Hebrank, A., and Chee, M. L. (2004). Cortical areas involved in object, background, and object-background processing revealed with functional magnetic resonance adaptation. J. Neurosci. 24, 10223-10228.

Gruppuso, V., Lindsay, D., and Kelley, C. M. (1997). The processdissociation procedure and similarity: defining and estimating recollection and familiarity in recognition memory. J. Exp. Psychol. Learn. Mem. Cogn. 23, 259-278.

Helmstaedter, C., Lendt, M., and Lux, S. (2001). VLMT: Verbaler Lern- und Merkfähigkeitstest. Göttingen: Beltz Test GmbH.

Henson, R. A., Hornberger, M., and Rugg, M. D. (2005). Further dissociating the processes involved in recognition memory: an fMRI study. J. Cogn. Neurosci. 17, 1058-1073.

Hesdorffer, D. C., Chan, S., Tian, H., Hauser, W. A., Dayan, P., Leary, L. D., and Hinton, V. J. (2008). Are MRI-detected brain abnormalities associated with febrile seizure type? Epilepsia 49, 765-771.

Hintzman, D. L., and Curran, T. (1994). Retrieval dynamics of recognition and frequency judgments: evidence for separate processes of familiarity and recall. J. Mem. Lang. 33, 1-18.
Hirtz, D. (2002). "Cognitive outcome of febrile seizures," in Febrile Seizures, eds T. Z. Baram and S. Shinnar (San Diego: Academic Press), 53-61.

Huang, C., and Chang, Y. (2009). The long-term effects of febrile seizures on the hippocampal neuronal plasticity - clinical and experimenta evidence. Brain Dev. 31, 383-387.

Hutchinson, J., Uncapher, M. R., and Wagner, A. D. (2009). Posterior parietal cortex and episodic retrieval: convergent and divergent effects of attention and memory. Learn. Mem. 16, 343-356.

Jessen, F., Flancke, S., Granath, D. O., Manka, C., Scheef, L., Papassotiropoulos, A., Schild, H. H., and Heun, R. (2001). Encoding and retrieval related cerebral activation in continuous verbal recognition. Brain Res. Cogn. Brain Res. 12, 199-206.

Johnson, J. D., Muftuler, L., and Rugg, M. D. (2008). Multiple repetitions reveal functionally and anatomically distinct patterns of hippocampal activity during continuous recognition memory. Hippocampus 18 975-980.

Kang, H. C., Burgund, E. D., Lugar, H. M., Petersen, S. E., and Schlaggar, B. L. (2003). Comparison of functional activation foci in children and adults using a common stereotactic space. Neuroimage 19, 16-28.

Kipp, K. H., Mecklinger, A., Becker, M. Reith, W., and Gortner, L. (2010). Infant febrile seizures: changes in declarative memory as revealed by event-related potentials. Clin. $\mathrm{Neu}$ rophysiol. 121, 2007-2016.

Kölfen, W., Pehle, K., and König, S. (1998). Is the long-term outcome of children following febrile convulsions favorable? Dev. Med. Child Neurol. 40, 667-671.

Lau, E., Phillips, C., and Poeppel, D. (2008). A cortical network for semantics: (de)constructing the N400. Nat. Rev. Neurosci. 9, 920-933.

Mecklinger, A. (2000). Interfacing mind and brain: a neurocognitive model of recognition memory. Psychophysiology 37, 565-582.

Mecklinger, A. (2010). The control of long-term memory: brain systems and cognitive processes. Neruosci. Biobehav. Rev. 34, 1055-1065.

Menon, V., Boyett-Anderson, J., and Reiss, A. (2005). Maturation of medial temporal lobe response and connectivity during memory encoding. Brain Res. Cogn. Brain Res. 25, 379-385.

Ment, L., Peterson, B., Vohr, B., Allan, W., Schneider, K., Lacadie, C., Katz, K., Maller-Kesselman, J., Pugh, K.,
Duncan, C., Makuch, R., and Constable, R. (2006). Cortical recruitment patterns in children born prematurely compared with control subjects during a passive listening functional magnetic resonance imaging task. J. Pediatr. 149, 490-498.

Nakayama, J. (2009). Progress in searching for the febrile seizure susceptibility genes. Brain Dev. 31, 359-365.

Nealis, J. G. T., Rosman, N. P., DePiero, T. J., and Ouellette, E. M. (1978) Neurologic sequelae of experimental febrile convulsions. Neurology 28, 246-250.

Ofen, N., Kao, Y.-C., Sokol-Hessner, P., Kim, H., Whitfield-Gabrieli, S. and Gabrieli, J. D. E. (2007). Development of the declarative memory system in the human brain. Nat. Neurosci. 10, 1198-1205.

Osterrieth, P. A. (1944). Le test de copie d'une figure complexe. Arch. Psychol. (Geneve) 30, 206-356.

Preston, A. R., Shrager, Y., Dudukovic, N. M., and Gabrieli, J. E. (2004) Hippocampal contribution to the novel use of relational information in declarative memory. Hippocampus 14, 148-152.

Quamme, J. R., Weiss, D. J., and Norman, K. A. (2010). Listening for recollection: a multivoxel pattern analysis of recognition memory retrieval strategies. Front. Hum. Neurosci. 4:61. doi:10.3389/fnhum.2010.00061

Ranganath, C., Yonelinas, A. P., Cohen, M. X., Dy, C. J., Tom, S. M., and D’Esposito, M. (2003). Dissociable correlates of recollection and familiarity within the medial temporal lobes. Neuropsychologia 42, 2-13.

Raven, J. C., Raven, J., and Court, J. H. (2002). Raven's Progressive Matrices und Vocabulary Scales. Frankfurt: Swets \& Zeitlinger B.V.

Rossion, B., and Pourtois, G. (2004). Revisiting snodgrass and vanderwart's object pictorial set: the role of surface detail in basic-level object recognition. Perception 33, 217-236.

Schweisthal, B. (1997). Die Leistungen von 7-bis 15 jährigen Kindern im Verbalen Lern- und MerkfähigkeitsTest (VLMT). Z. Neuropsychol. 8, 129-136.

Snodgrass, J. G., and Corwin, J. (1988). Pragmatics of measuring recognition memory: applications to dementia and amnesia. J. Exp. Psychol. Gen. 117, 34-50.

Sokol, D. K., Demyer, W. E., EdwardsBrown, M., Sanders, S., and Garg, B. (2003). From swelling to sclerosis: acute change in mesial hippocampus 
after prolonged febrile seizure. Seizure 12, 237-240.

Talairach, J., and Tournoux, P. (1988). Co-Planar Stereotyxic Atlas of the Human Brain. Stuttgart: Georg Thieme Verlag.

Tewes, U. (1997). Hamburg-WechslerIntelligenztest für Kinder - Revision 1983. Bern: Huber.

Tulving, E. (1972). "Episodic and semantic memory," in Organization of Memory, eds E. Tulving and W. Donaldson (New York: Academic Press), 381-403.

Van Landingham, K. E., Heinz, E. R., Cavazos, J. E., and Lewis, D. V. (1998). Magnetic resonance imaging evidence of hippocampal injury after prolonged focal febrile convulsions. Ann. Neurol. 43, 413-426.

Vargha-Khadem, F., Gadian, D. G., Watkins, K. E., Connelly, A., Van Paesschen, W., and Mishkin, M.
(1997). Differential effects of early hippocampal pathology on episodioc and semantic memory. Science 277, 376-380.

Vilberg, K. L., and Rugg, M. D. (2007). Dissociation of the neural correlates of recognition memory according to familiarity, recollection, and amount of recollected information. Neuropsychologia 45, 2216-2225.

von Zerssen, G., Mecklinger, A., Opitz, B., and von Cramon, D. (2001). Conscious recollection and illusory recognition: an event-related fMRI study. Eur. J. Neurosci. 13, 2148-2156.

Wais, P. E. (2008). fMRI signals associated with memory strength in the medial temporal lobes: a meta-analysis. Neuropsychologia 46, 3185-3196.

Yassa, M., and Stark, C. (2008). Multiple signals of recognition memory in the medial temporal lobe. Hippocampus 18, 945-954.

Yilmaz, D., Arhan, E., Yuksel, D., Ozcelik, A., Senbil, N., Serdaroglu, A., and Gurer, Y. K. (2008). Attitudes of parents and physicians toward febrile seizures. Clin. Pediatr. (Phila.) 47, 856-860.

Yonelinas, A. (2002). The nature of recollection and familiarity: a review of 30 years of research. J. Mem. Lang. 46, 441-517.

Yonelinas, A., Otten, L., Shaw, K., and Rugg, M. (2005). Separating the brain regions involved in recollection and familiarity in recognition memory. J. Neurosci. 25, 3002-3008.

Conflict of Interest Statement: The authors declare that the research was conducted in the absence of any commercial or financial relationships that could be construed as a potential conflict of interest.

Received: 15 July 2011; accepted: 28 January 2012; published online: 14 February 2012.

Citation: Kipp KH, Opitz B, Becker M, Hofmann J, Krick C, Gortner L and Mecklinger A (2012) Neural correlates of recognition memory in children with febrile seizures: evidence from functional magnetic resonance imaging. Front. Hum. Neurosci. 6:17. doi 10.3389/fnhum.2012.00017

Copyright (c) 2012 Kipp, Opitz, Becker, Hofmann, Krick, Gortner and Mecklinger. This is an open-access article distributed under the terms of the Creative Commons Attribution Non Commercial License, which permits non-commercial use, distribution, and reproduction in other forums, provided the original authors and source are credited. 